\title{
In vitro regeneration of gamma irradiated callus of artemisia annua and evaluation of increase artemisinin content by HPLC analysis
}

\begin{abstract}
Gamma radiations are the efficient ionizing radiations which have potential to induce mutations in plants. They also modify physiological characteristics of plant to create new mutants for large scale production of commercially important secondary metabolites. In present study, the effect of various low doses gamma irradiations $(5,10,15,20,25,30$ and 35Gy.) on growth, regeneration and elicitation of Artemisinin content from Artemisia аппиа was studied. Callus culture was established on Murashige and Skoog medium supplemented with phytohormones Napthalein acetic acid and N6-benzylaminopurine $\left(0.5 \mathrm{mgL}^{-1}\right)$. Effect of gamma doses on callus growth and elicitation of artemisinin content were investigated from M1T1, M2T2, M3T3, M4T4 and M5T5 generation of in vitro callus culture. In current study, it was found that low dose of gamma irradiations are effective strategy to enhance the yield of artemisinin in A. апnиа. The radiation sensitivity test was performed which is based on the reduction in survival rate of callus culture after treatment with gamma irradiation at different doses from 5 to $35 \mathrm{~Gy}$ which showed the negligible effect till $25 \mathrm{~Gy}$ but at 30 and $35 \mathrm{~Gy}$, it inhibited the callus growth and callus regeneration. The detection and quantification of artemisinin content was performed by HPLC analysis. The mobile phase for artemisinin elution was acetonitrile: water $(50: 50 \mathrm{v} / \mathrm{v})$ at a flow rate of $1 \mathrm{ml} \mathrm{min}{ }^{-1}$ and UV detection at $254 \mathrm{~nm}$. Artemisinin concentration of unknown sample was calculated by using the $Y$ equation $y=20266 x-5664 . R^{2}=0.914$ generated from standard curve. The retention time of artemisinin in above solvent system was observed at $7.3 \mathrm{~min}$. The higher accumulation of artemisinin content was observed in $15 \mathrm{~Gy}$ dose $7.04 \mu \mathrm{g} / \mathrm{gm}$ dry callus weight which is tenfold higher than non treated $0.700 \mu \mathrm{g} / \mathrm{gm}$ dry callus weight. The present study confirms that gamma radiation induced mutational changes in callus culture and it enhanced the artemisinin content.
\end{abstract}

Keywords: artemisia annua, artemisinin, antimalarial metabolite, induced mutations, gamma irradiation, callus culture, HPLC analysis
Volume 7 Issue 5 - 2018

\author{
Anita Patil,' Pooja Suryavanshi,' Devanand \\ Fulzele ${ }^{2}$ \\ 'Sant Gadge Baba Amravati University, India \\ 2Plant Biotechnology and Secondary Metabolites Section, \\ Nuclear Agriculture and Biotechnology Division, India
}

\begin{abstract}
Correspondence: Anita Patil, Sant Gadge Baba Amravati University, Amravati (M.S), 444 602, Topovan Road, India, Tel +91-
\end{abstract} 9881735354,Email anitapatil@sgbau.ac.in

Received: August 06, 2018| Published: October 05, 2018

\section{Introduction}

Artemisia annua is a aromatic annual herb also referred as sweet wormwood and called as ginghaosu which belongs to the family Asteraceae. ${ }^{1}$ According to published reports artemisinin content in A. аппиа vary in different parts and it has been detected in aerial parts such as leaves inflorescence, seeds and stem of plants. In leaves more artemisinin content is observed but ten fold higher is observed in flowers at full blooming stage. ${ }^{2,3}$ A. апnи a leaf contains peltate gland where high amount of artemisinin concentration is reported by many scientists. ${ }^{4}$ Professor You you Tu and coworker discovered artemisinin, the effective antimalerial drug in 1971 and got the nobel prize in Physiology or Medicine in 2015.5 A. annua contains sesquiterpenes lactone with endoperoxide bridge (C-O$\mathrm{C}-\mathrm{O}$ ) which is one of the most important drug in the treatment of chloroquine-resistant and cerebral malarias. ${ }^{6,7}$.Artemisinin and its derivatives (ARTs) are highly specific which can only be synthesized by $A$. апnиa. Other non volatile compounds which are sesquiterpene lactones origine are arteannuin B, artemisinic acid, artemisia methyl ester, artemisinol, artemisilactone, dihydroartemisinin, artesunate, artemether, and arteether. Among these, artemether and arteether get rapidly metabolized to dihydroartemisinin when enters the body and show strong anticancer activity. ${ }^{8}$ Mutational breeding involves high energy radiation such as $\mathrm{X}, \beta$ and $\gamma$-rays, which are electromagnetic radiations that initiate or inhibit the growth and differentiation of plant cells and organs. ${ }^{9}$ Gamma rays are the efficient ionizing radiations which induces high mutation in plants. They could also modify physiological characteristics of plant to create new mutants for production of high amounts of commercially important metabolites. ${ }^{10}$ Gamma rays interact with cells internal components and release free radicals. These free radicals either damage or modify the differentiation process, morphology, physiology and bioactive components depending on applied dose. ${ }^{11}$ Gamma irradiation are reported to enhanced the production of antioxidant defence enzymes and reduces ascorbic acid, total soluble protein, amino acids and sugars in callus cultures of Rosmarinus officinalis L. ${ }^{12}$ Low gamma dose gamma rays enhanced the growth of callus in Cucumis melo while higher dose reduced the growth. ${ }^{13}$ The gamma irradiation significantly stimulate the shikonin biosynthesis in Lithospermum erythrorhizon cell cultures. ${ }^{14}$ Artemisinin has very poor extinction coefficient. ${ }^{15,16}$ and poor staining characteristics also its analysis from natural sources has become a challenging task. The absence of specific chromophore in the molecule makes detection more difficult. ${ }^{17}$ Artemisinin shows the UV-Visible spectra at $254 \mathrm{~nm}$. Detection of artemisinin can be 
done by HPLC with UV, ELSD. ${ }^{18}$ and several GC methods with MS detection. The current study represents gamma irradiation to the callus culture in different doses 5, 10, 15, 20, 25, 30 and 35Gy and analysis of artemisinin content from gamma irradiated callus by HPLC. The main aims of the current study is to check the effect of gamma irradiation as an elicitor and analysis of increase artemisinin production in A. annua callus cultures.

\section{Material and methods}

\section{Collection of plant materials}

Artemisia annua variety CIM-Arogya received from CSIR-Central Institute of Medicinal and Aromatic Plants (CIMAP), Lucknow, India by Material transfer Agreement (MTA) between SGBAU and CIMAP.

\section{In vitro seed germination}

The seeds of $A$. апnиa was washed thoroughly with sterile distilled water. It was rinsed in $2 \%$ aqueous $\mathrm{HgCl}_{2}$ solution for $5 \mathrm{~min}$. Again it was washed with sterile distilled water three times. It was inoculated on Murashig and Skoog medium containing 2\% sucrose and are maintained at $24^{\circ} \mathrm{C}$ with $40 \% \mathrm{RH}$ (Relative humidity).

\section{Initiation of callus culture and media condition}

Leaves of size $(0.5-1.0 \mathrm{~cm}$ long) were excised from 4-week old in vitro germinated seedling and cultured on M.S. medium supplemented with $0.5 \mathrm{mg} / 1$ of NAA (Napthalin acetic) and BAP (N6Benzyl amino purine) and $2 \% \mathrm{w} / \mathrm{v}$ sucrose. The $\mathrm{pH}$ of the medium was adjusted at $6.8 \pm 0.2$. Culture medium was autoclaved at $121^{\circ} \mathrm{C}$ at $15 \mathrm{lbs}$ for $20 \mathrm{~min}$. Cultures were incubated at $24 \pm 1^{\circ} \mathrm{C}$ under $16 / 8 \mathrm{~h}$ light and dark conditions. The cultures were maintained on same media compositions and subcultured after every 4 weeks. After 5 subcultures callus cultures were used for further study.

\section{Gamma irradiation to callus cultures}

Callus induced from A. annua leaf was exposed to gamma irradiation for elicitation purpose at BARC, Mumbai. The 4week old callus were placed in irradiation chamber of the gamma cell and it was irradiated at different dose level of gamma irradiation $(5,10,15,20$, $25,30$ and $35 \mathrm{~Gy})$ at room temperature $\left(25 \pm 1^{\circ} \mathrm{C}\right)$. Gamma irradiation was performed using a gamma cell 4000 apparatus equipped with a ${ }^{60} \mathrm{Co}$ g-source (dose rate, $0.23 \mathrm{~Gy} / \mathrm{s}$ ) at Bhabha atomic research centre Trombay, Mumbai. The gamma-irradiated callus were labeled as G0M0 generation. After 25days of gamma irradiation the g-irradiated callus (M0T0) culture were subcultured on freshly prepared M.S. medium containing $2 \%$ sucrose and $0.5 \mathrm{mg} / \mathrm{L}$ Napthalein Acetic Acid (NAA) and N6 Benzyl Amino Purine (BAP). It was labelled as M1T1 generation of callus culture. Non treated callus were also kept and labelled as control. After M1T1 generation the same protocol was followed after 30days for subculture of M2T2, M3T3, M4T4 and M5T5 generation (Figure 1).

\section{Growth (biomass) measurement}

After completion of growth period (45days), the callus were removed from the medium and it was pressed on filter paper to remove excess water and weighed for the fresh weight. The same culture were dried in hot air oven at $55^{\circ} \mathrm{C}$ for $16 \mathrm{hr}$ and again it was weighed to obtain constant dry weight.

\section{Extraction of artemisinin}

In vitro callus of the M1T1, M2T2, M3T3, M4T4 and M5T5 generations were harvested and analyzed for the determination of artemisinin contents. Dried callus (100mg) were powdered in mortor and pistel with $1 \mathrm{ml}$ of petroleum ether and it was sonicated for $10 \mathrm{~min}$. The samples were centrifuged at $10,000 \mathrm{rpm}$ for 5 minutes and then supernatant was filtered by $0.2 \mu \mathrm{m}$ syringe filters (Axiva make $22 \mathrm{~mm}$ sterile filters) and transferred to clean glass vials and applied directly to high-performance liquid chromatography (HPLC) analysis.

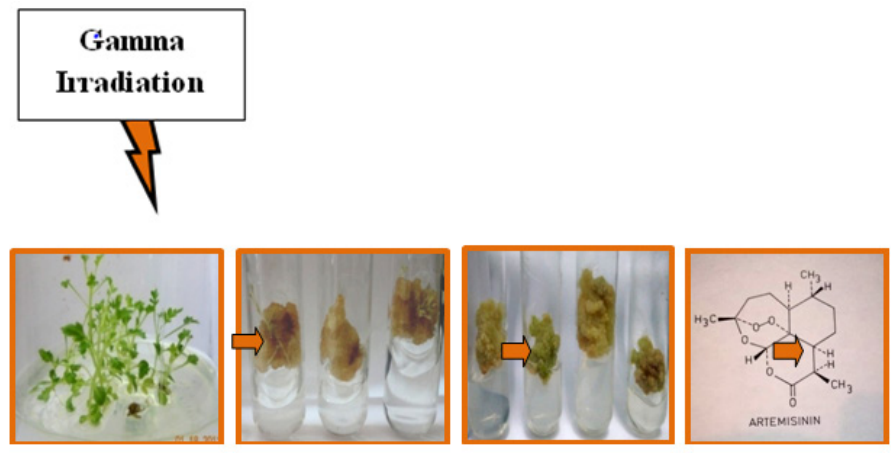

Figure I Diagramatic representation of induction of in vitro putative mutant of A.annua callus culture containing a high concentration of artemisinin after gamma irradiation.

Germinated plantlet Callus culture Putative mutant Artemisinin.

\section{Quantification of artemisinin by HPLC}

Artemisinin content was quantified by using isocratic analytical HPLC Agilent (1200 infinity series) equipped with an autosampler. The HPLC column was RP columns Eclipse Plus C18,4.6x100mm, 3.5 Make Agilent. The column oven was set at $25^{\circ} \mathrm{C}$. The mobile phase for artemisinin elution was acetonitrile: water $(50: 50 \mathrm{v} / \mathrm{v})$ at a flow rate of $1 \mathrm{mlmin}^{-1}$ and UV detection at $254 \mathrm{~nm} .1 \mathrm{mgml}^{-1}$ stock solution of standard artemisinin was prepared in methanol. For the calibration curve, $20-100 \mu l$ of standard solution of artemisinin was applied in triplicate onto the HPLC. The peak areas were recorded and a calibration curve of artemisinin was prepared by plotting peaks area against concentrations. Calibration graph was prepared from different concentration of standard of Artemisinin and concentration of unknown sample was calculated by using the $Y$ equation $y=20266 x$ - 5664. $R^{2}=0.914$ generated from standard curve. The retention time of artemisinin in above solvent system observed at $7.3 \mathrm{~min}$.

\section{Results and discussion}

\section{Radiation sensitivity test}

The radiation sensitivity test were based on the survival rate of callus cultures treated with different doses of gamma irradiation, in comparison with non-irradiated callus culture which have $100 \%$ survival rate. In the current study we observed that the survival rate of gamma irradiated callus was $100 \%$ which resembles with the results obtained by. ${ }^{19}$ They reported $100 \%$ in vitro survival of Dracaena surculosa plants at low doses of gamma irradiation and the survival rate decreased at high doses of gamma irradiation. Likewise, increasing the dose of gamma irradiation showed negative effect on the survival of callus and plant regeneration..$^{20}$ The survival rate of gamma irradiated callus was decreased with increased doses of gamma 
irradiation. However, all irradiated callus at various doses showed $100 \%$ survival, while callus cultures treated with higher doses in the range from 30 to $35 \mathrm{~Gy}$ showed the less survival rate compared with low dose treatment. Whereas callus subjected to exposure at 5 to $25 \mathrm{~Gy}$ showed significant survival rates during M1T1 to M5T5 generation. These results were in accordance with the radiation sensitivity test performed by ${ }^{21,22}$ they reported that gamma doses decreased the survival percentage of plantlets of Orthosiphon stamineus.

\section{Effects of gamma irradiation on regeneration of callus and callus size during MITI to M5T5 generation}

The callus culture initiated from the gamma irradiated callus treated with different irradiation doses 5, 10, 15, 20, 25,30 and 35Gy. Initially a steady decline in the callus growth and callus regeneration in M1T1 generation was observed. Whereas in subsequent generation from M2T2 to M5T5 at 5, 10 and 15Gy the maximum callus regeneration and callus growth of was observed.

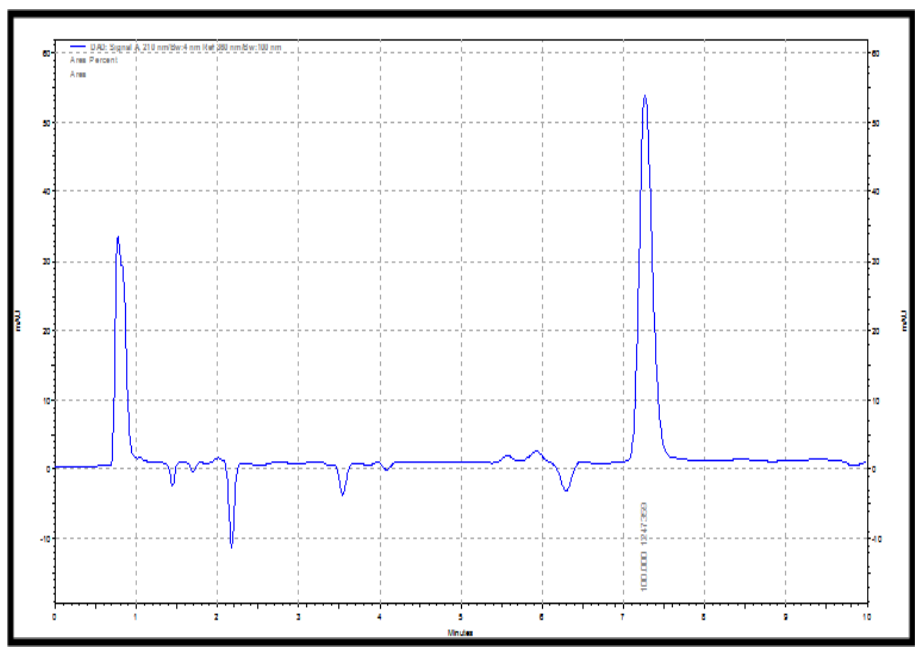

Figure 2 HPLC analysis showing the presence of artemisinin in Artemisia annua extracts.

Non-irradiated callus showed the maximum callus size $1.59 \mathrm{~cm}$, whereas irradiated callus at $5 \mathrm{~Gy}$ showed $1.69 \mathrm{~cm}$ size in $1 \mathrm{month}$, some abnormalities were found during the growth and regeneration of callus treated in the range of 30 and $35 \mathrm{~Gy}$ that the callus of these two generation showed stunted growth, less biomass and the colour change was observe from green to browinish black as compared to other low doses. The results of current research showed that high-gamma doses reduced regeneration of callus and formed stunted growth, whereas low doses improved the callus regeneration as compared to nonirradiated callus (Figure 2). The results in the present study were supported by ${ }^{19}$ they reported that gamma irradiation at low doses increased the number of shoot cultures of Dracaena surculosa. Also, similar observation was reported by. ${ }^{8}$ The efficiency of shoot growth showed variation when exposed to low doses of gamma irradiation in the range from 5 to $40 \mathrm{~Gy}$. A significant stimulation of growth rate was observed at low doses in the range from 5 to $20 \mathrm{~Gy}$ and after that the growth was drastically reduced when exposed to high doses from M1T1to M5T5 generation. Maximum biomass was obtained when the callus were exposed to the $5 \mathrm{~Gy}$ dose as compared to non-irradiated callus cultures. In the present study, low gamma doses favored callus growth and formed $3.3 \mathrm{~g} \mathrm{FW}$ biomass at $5 \mathrm{~Gy}$, which is more than non- irradiated callus cultures which was $2.8 \mathrm{gm}$ FW. However, biomass $0.25 \mathrm{gm}$ and $0.27 \mathrm{~g} \mathrm{FW}$ obtained from callus irradiated at 30 and $35 \mathrm{~Gy}$. In addition, some morphological abnormalities were observed in the irradiated callus cultures at high doses that showed some brownish coloured pigmentation when compared with non irradiated callus cultures. Improvement of secondary metabolites by using low doses of gamma irradiation has previously reported by several researchers. ${ }^{23}$ recently reported that at $20 \mathrm{~Gy}$ dose of gamma irradiation induces cell growth and achieved $0.098 \%$ dry weight camptothecin and $0.0043 \%$ dry weight 9-methoxy camptothecin which is 20 -fold more than nonirradiated callus cultures of Nothapodytes foetida.$^{24}$ reported that cell cultures of Orthosiphon stamineus when exposed to low doses the growth rate was not affected and it showed the high increase in fresh weight, whereas cultures exposed at higher doses showed the less increase in fresh weight. Earlier many researchers have investigated that treatment with high gamma irradiation doses was harmful, whereas low doses showed positive effects. As observed in the current study, it has been reported by Ling et al. ${ }^{25}$ that plant growth increased at low doses in Citrus sinensis, whereas growth was inhibited when radiation dose increased. Similar results have been demonstrated by Kangarasu et al. ${ }^{26}$ that there is reduction in the survival percentage of Manihot esculenta crantz stem cuttings with the increased doses of gamma irradiation. ${ }^{27}$ reported that high doses of gamma rays showed reduced and stunted leaves and reduced plant heights of Picea abies,Larix deciduas and Pinus sylvestris, whereas low doses of gamma rays showed most positive effects on growth. According to ${ }^{28}$ high doses of gamma irradiation caused inhibition of plant tissue and failure of RNA synthesis inturn protein synthesis. Gamma rays belongs to ionization radiation, which generates free radicals and high doses posses harmful effects on plant morphology, anatomy, biochemistry, and physiology which depends on the irradiation dose. Gamma rays are the mutagen with high radiation energy which leads to damage the covalent bond, hydrogen bond of biomolecules in the cell and may cause the chromosome damage and finally cell death (Figure 3). ${ }^{29}$

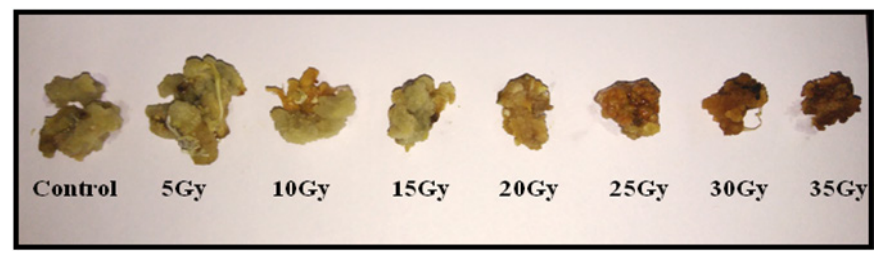

Figure 3 The phenotypes of the control and callus cultures of A.annua exposed to different doses of gamma irradiation: I-month cultures after gamma irradiation treatment and the effects of low doses of gamma irradiation on growth of A.annua callus cultures on MS medium supplemented with Napthalein acetic acid (NAA) and N6 Benzyl amino purine (BAP) $\left(0.5 \mathrm{mg} \mathrm{L}^{-1}\right)$ during the MITI generation.

\section{Effects of gamma irradiation on artemisinin production during $\mathrm{mlt} \mathrm{l}$ to $\mathrm{m} 5 \mathrm{t} 5$ generations}

The increase production of value added commercial products through plant cell culture has been a challenging task for many researchers (Figure 4). Artemisinn is the sesquiterpenes lactone with endoperoxide bridge which is highly volatile in nature and hard to produce on large scale from $A$. annua. Thus, to enhance the production of artemisinin and selection of a putative mutant using gamma-irradiated callus was subsequently subjected to the 
estimation of artemisinin during different generations. By comparing the production, significant variation has been observed during the successive five in vitro subcultures (M1T1, M1T2, M1T3, M4T4 and M5T5 generations) and it was found that low doses of gamma irradiation enhanced the production of artemisinin (Figure 5). Data in Figure 5 showed that maximum artemisinin production of $7.02 \mu \mathrm{g} / \mathrm{gm}$ dry callus weight was recorded in $15 \mathrm{~Gy}$ callus of M2T2 generation which is tenfold higher than non irradiated callus, whereas in non irradiated callus culture theminimum concentration of $0.70 \mu \mathrm{g} /$ dry weight of callus was obtained. HPLC was performed after the irradiation and subsequent subcultures and it showed that the level of artemisinin in the treated callus cultures was higher than the non treated callus in each generation. However, the artemisinin concentration of treated callus was increased during M1T1, M2T2, M3T3, M4T4 and M5T5 generations and it becomes higher than nonirradiated callus cultures. ${ }^{30}$ Reported that shoot cultures regenerated from irradiated callus of Artemisia cina contain high artemisinin content than non irradiated plants. They found the highest artemisinin content was $5 \mathrm{mg} / \mathrm{g}$ in mutant line which also have widest leaf area compared to non treated plant. ${ }^{31}$ reported recently that the yield of shikonin derivatives increased by $180-400 \%$ using low-dose gammairradiation has been observed in callus cultures of Lithospermum erythrorhizon. ${ }^{32}$ There has been no information reported in the literature on using this technology to enhance the artemisinin content through callus culture in A. аnnua. So, this is the first report showing variation of artemisinin content in the population of gamma irradiated callus. The experimental results showed that the accumulation of artemisinin in irradiated callus was not constant in all the generations. The results obtained from current study showed achievement of a putative mutant, which contained high amounts of artemisinin as compared to non irradiated ones and large-scale production can be obtained via suspension culture. The study confirms that the free radicals generated in experimental plants after gamma irradiation may elicit stress responses in cell cultures and therefore variations in yield were obtained at different doses of gamma irradiation. According to the results, obtained from the current study could suggest that low doses of gamma irradiation shows promising effect to achieve putative mutants with high concentrations of artemisinin (Figure 6).

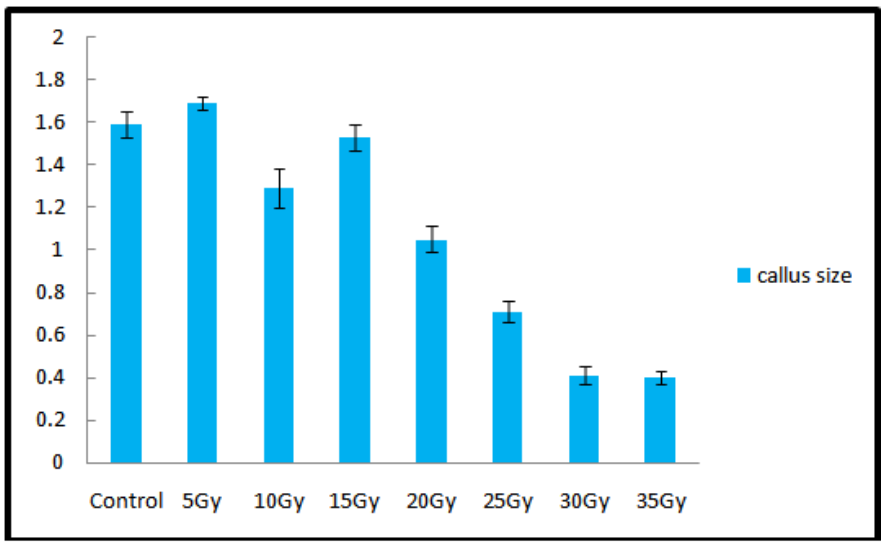

Figure 4 Effects of low doses of gamma irradiation on callus size and regeneration of A.annua on MS medium supplemented with Napthalein acetic acid and N6-benzylaminopurine $\left(0.5 \mathrm{mg} \mathrm{L}^{-1}\right)$. The observation was obtained from triplicate determinations Mean $( \pm \mathrm{SE})$.

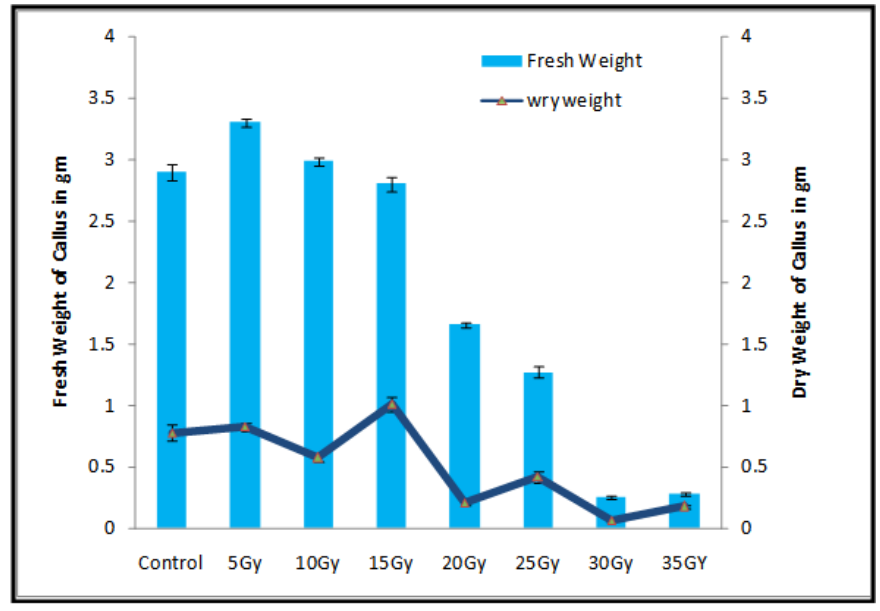

Figure 5 Effects of low doses of gamma irradiation on callus fresh weight and dry weight. The observation was obtained from triplicate determinations Mean $( \pm S E)$.

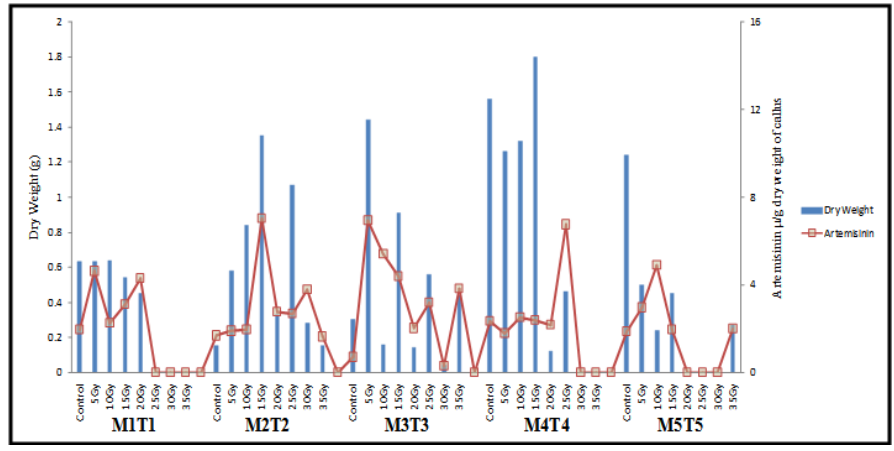

Figure 6 Effect of low doses of gamma irradiation on callus size and accumulation of artemisinin in callus cultures of Artemisia annua on MS medium supplemented with Napthalein acetic acid and These results were representative from three independent replicates $( \pm S E)$.

\section{Conclusion}

The current study conclude that low doses of gamma irradiation proven to be ecofriendly and a low cost method that can be used for the efficient selection of mutants to enhance the artemisinin production and other secondary metabolites. In the present study, it was found that a low dose of gamma irradiation are effective strategy to enhance the yield of artemisinin in A.аnпиa. The radiation sensitivity test was based on the reduction in survival rate of callus culture after treatment with gamma irradiation at different doses from 5 to $35 \mathrm{~Gy}$ which showed the negligible effect till $25 \mathrm{~Gy}$ but at 30 and $35 \mathrm{~Gy}$, it inhibited the callus growth and callus regeneration. The higher accumulation of artemisinin content was observed in $15 \mathrm{~Gy}$ dose $7.04 \mu \mathrm{g} / \mathrm{gm}$ dry callus weight which is ten fold higher than non treated $0.700 \mu \mathrm{g} / \mathrm{gm}$ dry callus weight of callus. Our results have estimated a high-yielding putative mutant, which contained high amounts of artemisinin content through callus culture and we are beginning towards the large scale production of callus through suspension culture for high artemisinin production. 


\section{Future scope}

The results obtained from the current study proved to be encouraging for future research on the selection of mutant after gamma irradiation at various doses which enhanced the yield of artemisinin from A. аппиа.

\section{Acknowledgements}

This work was supported by DAE, BRNS, Govt of India. Author is thankful to the Department of Atomic Energy, Board of Research in Nuclear Sciences, Mumbai, India for providing financial support under major research project Sanction letter No. 35/14/30/2016BRNS/35106

\section{Conflict of interest}

The author declares no conflict of interest.

\section{References}

1. Weng CW. The forest of china, with a survey of grass land and dessert vegetations. 1st ed. Harward University Maria Moors Cobot Foundation; 1961. 5:171-187.

2. Tellez MR, Canel C, Rimando AM, et al. Differential accumulation of isoprenoids in glanded and gland-less Artemisia annua L. Photochemistry. 1999;52(6):1035-1040.

3. Ferreira JFS, Janick J. Floral morphology of Artemisia аппиа with special reference to trichomes. International Journal of plant science. 1995;156(6):807-815

4. Chunzhao Liu, Yan Zhao, Yuchun Wang. Artemisinin: current state and perspectives for biotechnological production of an antimalarial drug. Applied Microbiology and Biotechnology. 2006;72(1):11-20.

5. Zongru Guo. The modification of natural products for medical use. Acta Pharmaceutica Sinica B. 2017;7(2):119-136.

6. Liu JM, Ni MY, Fan JF, et al. Structure and reaction of arteannuin. Acta Chimica Sinica. 1979;37(2):120-143.

7. LiZQ, Liu Y, Liu BYet al. Cloning, E. coli expression and molecular analysis of amorpha-4,11-diene synthase from a high-yield strain of Artemisia апnи L. Journal of Integrated Plant Biology. 2006;48(12):1486-1492.

8. Habibi Z, Ghanian S, Ghasemi S, et al. Chemical composition and antimicrobial activity of the volatile oil from seeds of Artemisia annua $\mathrm{L}$. from Iran. Natural product research. 2013;27(2):198-200.

9. Hasbullah NA, Taha RM, Saleh A, et al. Irradiation effect on in vitro organogenesis callus growth and plantlet development of Gerbera jamesoni. Horticultura Brasileira. 2012;30(2):252-257.

10. Naito K, Kusaba M, Shikazono N, et al. Transmissible and nontransmissible mutations induced by irradiating Arabidopsis thaliana pollen with (gamma)-rays and carbon ions. Genetics. 2005;169(2):881-889.

11. Cheema M, Qamar R (2003) Effect of gamma rays on M1 generation in Basmati rice. Pakistan Journal of Botany. 2003;35(5):791-795.

12. El-Beltagi HS, Ahmed OK, El-Desouky W. Effect of low doses g-irradiation on oxidative stress and secondary metabolites production of rosemary (Rosmarinus officinalis L.) callus culture. Radiation physics and Chemistry. 2011;80(9):968-976.

13. Venkateshwarlu M. Effect of gamma rays on different explants of callus treatment of multiple shoots in Cucumis melo cv. Bathasa. Journal of Environmental Biology. 2008;29(5):789-792.
14. Wi SG, Chung BY, Kim JH, et al. Ultrastructural changes of cell organelles in arabidopsis stems after gamma irradation. Journal of Plant Biology. 2005;48(2):195-200.

15. Ferreira J, Gonzalez JM. Analysis of underivatized Artemisinin and related sesquiterpene lactones by High-Performance Liquid Chromatography with ultraviolet detection. phytochemical Analysis. 2009;20(2):91-97.

16. Alexei AL, Adam W, Neil S, et al. Development of HPLC analytical protocols for quantification of Artemisinin in biomass and extracts. Journal of Pharmaceutical and Biomedical Analysis. 2009;49(4):908-915.

17. Abdul M, Ibrar A, Waheed A, et al. Survey of Artemisinin Production by Diverse Artemisia Species in Northern Pakistan. Malaria Journal. 2010;9:310.

18. Bharathi A, Yan-Hong W, Troy JS, et al. Comparison of LC-UV, LCELSD, and LC-MS methods for the determination of sesquiterpenoids in various species of Artemisia. Chromatographia. 2009;70(56):797-803.

19. Sakr SS, El-Khateeb MA, Taha HS, et al. Effect of gamma irradiation on in vitro growth chemical composition and anatomical structure of Dracaena surculosa L. Journal of applied science Research. 2013;9:3795-3801.

20. Hossain MF, Alam MS. Pakistan Journal of Biological Science. 2001;4:670-671.

21. Kiong ALP, Lai AG, Hussein S, et al. Physiological responses of Orthosiphon stamineus plantlets to gamma irradiation. Am-Eurasian Journal of Sustainable Agriculture. 2008;2:135-149.

22. Kapare V, Satdive R, Fulzele P, et al. Impact of Gamma Irradiation Induced Variation in Cell Growth and Phytoecdysteroid Production in Sesuvium portulacastrum. Journal of Plant Growth and Regulators. 2017;36(4):919930.

23. Sipahimalani AT, Fulzele DP, Heble MR. Rapid Method for the Detection and Determination of Artemisinin by Gas Chromatography. Journal of Chromatography. 1991;538(2):452-455.

24. Ling A, Ong A, Hussein S, et al. Morphological and physiological responses of Orthosiphon stamineus callus to gamma irradiation at different doses. World Journal of Agriculture Science. 2010;6:58-66.

25. Ling A, et al. Physiological Responses of Citrus sinensis to gamma irradiation. World Applied Science Journal. 2008;5:12-19.

26. Kangarasu S, Ganshram S, John Joel A. Determination of lethal dose for gamma rays and ethyl methyl sulphonate induced mutagenesis in cassava (Manihot esculenta crantz). International journal of Science research. 2014;3:2-6

27. Smelkova L. Effect of gamma rays on the germination of conifer seeds. Acta fac for Zvolen Slovak. 1999;41:81-90.

28. Bajaj YPS. Effect of gamma-irradiation on growth, RNA, protein, and nitrogen contents of bean callus cultures. Ann Bot. 1970;34:1089-1096.

29. Vonarx EJ, Mitchell HL, Karthikeyan R, et al. DNA repair in higher plants Mutation Research. 1998;400:187-200.

30. Aryanti. Production of artemisinin in shoot cultures of Artemisia cina irradiated callus. Majalah Farmasi Indonesia. 2010;21(1):27-31.

31. Koobkokkruad T, chochai A, Kirdmanee C, et al. Effects of low-dose gamma irradiation on artemisinin content andamorpha-4,11-diene synthase activity in Artemisia annua L. International Journal of Radiation Biology. 2008;84(11):878-884.

32. Chung BY, Lee YB, Baek MH, et al. Effects of low-dose gammairradiation on production of shikonin derivatives in callus cultures of Lithospermum erythrorhizon S. Radiation Physics and Chemistry. 2006;75(9):1018-1023. 